\title{
Clinical validation of non-invasive hemoglobin monitoring-a prospective observational study
}

\author{
Reinhard Hahn, MD (1) - Aylin Bilir · Ulrike Weber, PhD, MD • \\ Oliver Kimberger, MD,MSc $\cdot$ Stephan Kettner, MD
}

Received: 2 March 2020/Revised: 25 November 2020/Accepted: 25 November 2020/Published online: 12 February 2021

(C) Canadian Anesthesiologists' Society 2021

\section{To the Editor,}

Measurement of hemoglobin is a requirement for many patients in emergency and intensive care medicine. Avoiding the side effects of anemia and transfusion is a perpetual challenge. ${ }^{1,2}$ A continuous non-invasive hemoglobin monitoring would be helpful to facilitate strict transfusion protocols in patients at risk for active bleeding. The Rainbow SET ${ }^{\circledR}$ Radical-7 Pulse COOximeter (Masimo, Irvine, CA, USA) (MR7) enables continuous non-invasive hemoglobin monitoring. Following approval of the Ethik-Kommission der Medizinischen Universität Wien (13 April 2010), we conducted a prospective observational study testing the MR7 in 100 patients undergoing orthopedic surgery and the potential need for transfusion of packed red blood cells. We compared measurements from the MR7 to those from the ABL 700 point-of-care blood gas analyser (Radiometer $\mathrm{GmbH}$, Copenhagen, Denmark).

The mean and 95\% limits of agreement (LOA) between the methods were calculated using the Bland-Altman method, ${ }^{3}$ and association of measurements were assessed using Pearson's correlation. Three subgroups were defined by the hemoglobin values measured by the blood gas analyser: Group Hb7-10 (7 - $\left.9.9 \mathrm{~g} \cdot \mathrm{dL}^{-1}\right)$, Group Hb10-13 $\left(10-12.9 \mathrm{~g} \cdot \mathrm{dL}^{-1}\right)$, and Group $\mathrm{Hb}>13\left(>13 \mathrm{~g} \cdot \mathrm{dL}^{-1}\right)$.

To account for multiple measurements in individual patients, a random effects model was used to calculate the LOA of the Bland-Altman analysis. We defined a

R. Hahn, MD $(\varangle) \cdot$ A. Bilir · U. Weber, PhD, MD .

O. Kimberger, MD,MSc $\cdot$ S. Kettner, MD

Department of Anaesthesia, Critical Care and Pain Medicine,

Medical University of Vienna, Vienna, Austria

e-mail: reinhard.hahn@meduniwien.ac.at clinically acceptable error with a LOA of less than \pm 2 $\mathrm{g} \cdot \mathrm{dL}^{-1}$. Additionally, the percentage of non-valid measurements, the number of probes, and the time needed was recorded. The continuous non-invasive hemoglobin measurement by Masimo $(\mathrm{SpHb})$ is defined to be valid if the value is within the predefined range $( \pm 2$ $\mathrm{g} \cdot \mathrm{dL}^{-1}$ ) compared with the standard point-of-care laboratory blood gas analysis (HbLab).

The following data are reported as median [interquartile range (IQR)]. It took 17 [9-34] $\mathrm{min}$ and 22 [7-70] min under clinical conditions to have reliable measurements or a clear failure, respectively. We used 112 sensors (version R2-25, Rev E) in 100 patients, and 12 sensors did not function properly. Valid paired hemoglobin measurements were available in 91 patients, which provided 557 paired measurements. The median [IQR] of paired measurements per patient was 5 [4-8].

The Bland-Altman plot comparing $\mathrm{SpHb}$ and HbLab is shown in Figure A. Overall, the MR7 overestimated the hemoglobin with a mean (standard deviation [SD]) of 0.02 (1.73) $\mathrm{g} \cdot \mathrm{dL}^{-1}$ with a $95 \% \mathrm{LOA}$ of $-3.80 \mathrm{~g} \cdot \mathrm{dL}^{-1}$ to 3.78 $\mathrm{g} \cdot \mathrm{dL}^{-1}$. The MR7 overestimated the hemoglobin with a mean (SD) of $0.62(1.78) \mathrm{g} \cdot \mathrm{dL}^{-1}$ in the $\mathrm{Hb}>13$ group, while underestimating the hemoglobin in the Hb7-10 group and $\mathrm{Hb} 10-13$ group, with a mean (SD) of -0.16 (1.56) $\mathrm{g} \cdot \mathrm{dL}^{-1}$ and $-0.15(1.73) \mathrm{g} \cdot \mathrm{dL}^{-1}$, respectively. The Pearson correlation coefficient comparing $\mathrm{SpHb}$ and $\mathrm{HbLab}$ measurements was $0.67(P<0.001)$.

The ability to track changes in hemoglobin over time is equally as important as the absolute agreement of $\mathrm{SpHb}$ with the HbLab. Trend analysis was performed with a fourquadrant plot (Figure B) as suggested by Critchley et al. ${ }^{4}$ Concordance of all measurements was 53\% and 57\% if the $20 \%$ of measurements within $0.5 \mathrm{~g} \cdot \mathrm{dL}^{-1}$ were excluded. 

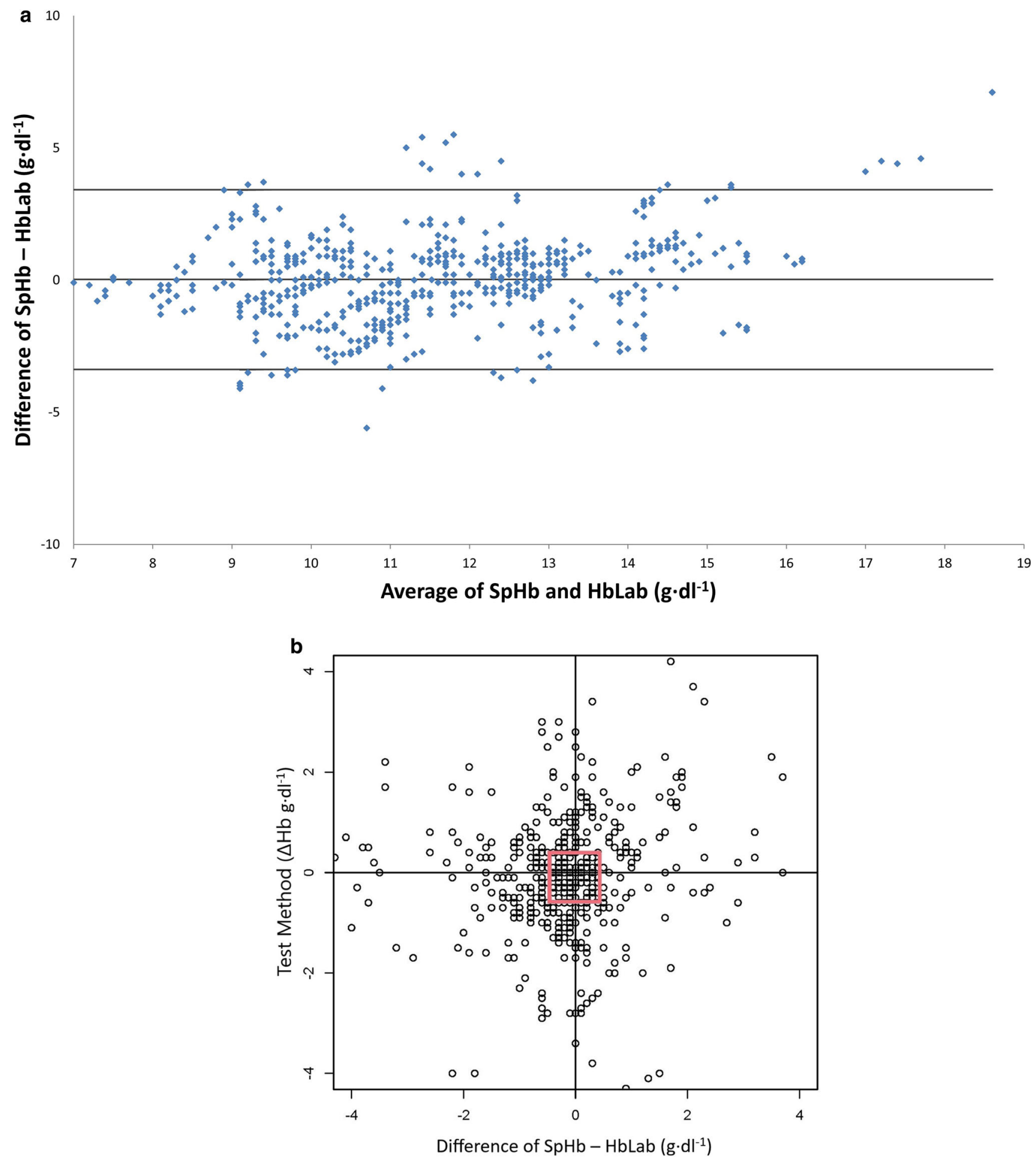

Figure A) Bland-Altman plot of the difference between continuous non-invasive hemoglobin measurement (SpHb) and arterial hemoglobin measured by a point-of-care laboratory blood gas analyzer ( $\mathrm{HbLab}$ ) against the average of $\mathrm{SpHb}$ and $\mathrm{HbLab}$. The horizontal lines correspond to the bias and limits of agreement (LOA). B) The four-quadrant plot displays the trends of the reference method (HbLab) versus the test method $(\mathrm{SpHb})$. Concordance rates without data in the exclusion zone was $57 \%$ (exclusion zone $0.5 \mathrm{~g} \cdot \mathrm{dL}^{-1}$ containing $20 \%$ of data—bold line) and $53 \%$ including all data. The model took into account repeated measurements in the same patients using random effects. 
Our results contrast those of Adel et al., ${ }^{5}$ who reported excellent trending ability of the MR7.

Given the wide limits of agreement between $\mathrm{SpHb}$ and $\mathrm{HbLab}$, probe failure in nearly $10 \%$ of cases, and relatively poor tracking, we believe the MR7 to be insufficiently reliable to guide transfusion decisions in the clinical setting.

Disclosures None.

Funding statement Departmental sources.

Editorial responsibility This submission was handled by Dr. Gregory L. Bryson, former Deputy Editor-in-Chief, Canadian Journal of Anesthesia.

\section{References}

1. Shander A, Javidroozi M, Ozawa S, Hare GM. What is really dangerous: anaemia or transfusion? Br J Anaesth 2011; 107(Suppl 1): i41-59.

2. Jandu AS, Vidgeon S, Ahmed N. Anaemia and transfusion triggers in critically ill patients - what we have learnt thus far. J Intensive Care Soc 2019; 20: 284-9.

3. Bland JM, Altman DG. Measuring agreement in method comparison studies. Stat Methods Med Res 1999; 8: 135-60.

4. Critchley LA, Lee A, Ho AM. A critical review of the ability of continuous cardiac output monitors to measure trends in cardiac output. Anesth Analg 2010; 111: 1180-92.

5. Adel A, Awada W, Abdelhamid B, et al. Accuracy and trending of non-invasive hemoglobin measurement during different volume and perfusion statuses. J Clin Monit Comput 2018; 32: 1025-31.

Publisher's Note Springer Nature remains neutral with regard to jurisdictional claims in published maps and institutional affiliations. 